\section{Lack of adjunctive benefit of Er:YAG laser in non-surgical periodontal treatment: a randomized split-mouth clinical trial}

Rotundo R, Nieri M, Cairo F, Franceschi D, Mervelt J, Bonaccini D, Esposito M, Pini-Prato G. Lack of adjunctive benefit of Er:YAG laser in non-surgical periodontal treatment: a randomized split-mouth clinical trial. J Clin Periodontol 2010; 37: 526-533. doi: 10.1111/j.1600-051X.2010.01560.x.

\begin{abstract}
Aim: This split-mouth, randomized, clinical trial aimed to evaluate the efficacy of erbium-doped:yttrium-aluminium-garnet (Er:YAG) laser application in non-surgical periodontal treatment.

Materials and Methods: A total of 27 patients underwent four modalities of nonsurgical therapy: supragingival debridement; scaling and root planing (SRP) +Er:YAG laser; Er:YAG laser; and SRP. Each strategy was randomly assigned and performed in one of the four quadrants. Clinical outcomes were evaluated at 3 and 6 months. Subjective benefits of patients have been evaluated by means of questionnaires. Results: Six months after therapy, Er:YAG laser showed no statistical difference in clinical attachment gain with respect to supragingival scaling $[0.15 \mathrm{~mm}(95 \% \mathrm{CI}$ $-0.16 ; 0.46)]$, while SRP showed a greater attachment gain than the supragingival scaling [0.37 mm (95\% CI 0.05; 0.68)]. No difference resulted between Er:YAG laser+SRP and SRP alone $[0.05 \mathrm{~mm}(95 \% \mathrm{CI}-0.25 ; 0.36)]$.

Conclusions: The adjunctive use of Er:YAG laser to conventional SRP did not reveal a more effective result than SRP alone. Furthermore, the sites treated with Er:YAG laser showed similar results of the sites treated with supragingival scaling.
\end{abstract}

Roberto Rotundo ${ }^{1}$, Michele Nieri ${ }^{1}$, Francesco Cairo ${ }^{1}$, Debora Franceschi ${ }^{1}$, Jana Mervelt ${ }^{1}$, Daniele Bonaccini ${ }^{1}$, Marco Esposito ${ }^{2,3}$ and Giovanpaolo Pini-Prato ${ }^{1}$

${ }^{1}$ Department of Periodontology, University of Florence, Firenze, Italy; ${ }^{2}$ Department of Oral and Maxillofacial Surgery, School of Dentistry, The University of Manchester, Manchester, UK; ${ }^{3}$ Department of Biomaterials, The Sahlgrenska Academy at Göteborg University, Göteborg, Sweden This paper was the winner of the 2009 Jaccard
Prize competition.

Key words: laser; non-surgical/mechanical; periodontitis; scaling/root planing; treatment/ therapy

Accepted for publication 3 February 2010
The primary aim of non-surgical periodontal treatment is to arrest disease progression by eliminating bacterial infection, to reduce soft tissue inflammation and to re-attach periodontal tissues to the root surface infected

\section{Conflict of interest and source of funding statement}

The authors declare that they have no conflict of interests.

The study was self-funded by the authors and/or their institution. previously (Slots 1979, Daeveau et al. 1997, Slots \& Ting 1999).

Root debridement, consisting of the elimination of plaque, calculus and bacterial debris from the root surface is generally accomplished by hand or power-driven instruments, which have been shown to be equally effective (Tunkel et al. 2002, Walmsley et al. 2008). However, the main limitation of conventional non-surgical therapy is the difficult access in areas such as furcations, grooves, concavities and deep pockets (Bower 1979, Cobb 1996).
In the last decades, laser therapy has been proposed as an alternative or adjunct to conventional non-surgical therapy, due to its capability to obtain tissue ablation, haemostatic, bactericidal and detoxification effects against periodontal pathogens (Schultz et al. 1986, Folwaczny et al. 2002, Aoki et al. 2004, Ishikawa et al. 2004, Schwarz et al. 2008). Among the different types of laser, erbium-doped:yttrium-aluminium-garnet (Er:YAG) laser appears to be the most suitable to be used for periodontal treatment, due to its ability of both soft 
and hard tissue ablation. Unlike carbon dioxide $\left(\mathrm{CO}_{2}\right)$ and neodymium-doped: yttrium-aluminium-garnet (Nd:YAG) lasers, which produce carbonization and major thermal side effects when used on hard tissues such as root surface and bone (Tewfik et al. 1994, Wilder-Smith et al. 1995, Israel et al. 1997), the Er:YAG laser results in very little heat generation (Aoki et al. 2004).

Despite in vitro studies (Tewfik et al. 1994, Wilder-Smith et al. 1995, Israel et al. 1997) have demonstrated its ability to create a surface that suggests biocompatibility for soft tissue attachment, few clinical trials have been conducted until now evaluating the clinical outcomes of Er:YAG laser in mechanical periodontal treatment (Schwarz et al. 2001, 2003a, b). In a randomized controlled clinical trial (Lopes et al. 2008), it was reported that conventional scaling and root planing (SRP), SRP+Er:YAG laser and Er:YAG laser alone showed a greater pocket depth reduction than the no treatment group. No significant differences were observed among SRP+Er:YAG laser, Er:YAG laser alone, SRP and no treatment groups in terms of clinical attachment gain and gingival recession.

A recent systematic review (Schwarz et al. 2008) reported that Er:YAG laser application in non-surgical periodontal therapy compared with mechanical debridement resulted in similar clinical outcomes, both in the short and the long term (up to 24 months), in patients with chronic periodontitis. However, due to the high heterogeneity of the studies, a meta-analysis could not be performed, and therefore their conclusions are based on a simple narrative synthesis.

Another systematic review (Karlsson et al. 2008) that considers all types of lasers showed a weak evidence to support the efficacy of laser treatment as an adjunct to conventional non-surgical therapy in the application of either $\mathrm{CO}_{2}$, Nd:YAG, neodymium-doped:yttriumaluminium-perovskite, erbium-doped: yttrium-scandium-gallium-garnet or different diode laser wavelengths. The authors concluded that further RCTs are needed.

The Consensus Report of the Sixth European Workshop on Periodontology (Sanz et al. 2008) suggested that welldesigned randomized controlled clinical trials using a larger number of patients in order to further evaluate Er:YAG laser application when compared with conventional mechanical debridement are needed. An indication for a well- designed RCTs set-up may be the use of the CONSORT (CONsolidated Standards of Reporting Trials) statement (Moher et al. 2001).

The aim of this split-mouth randomized clinical trial is to evaluate according to the CONSORT statements the efficacy of four different non-surgical approaches (supragingival debridement, and a combination of Er:YAG laser with SRP, Er:YAG laser, SRP alone) at 6 months, in patients with moderate to advanced chronic periodontitis.

\section{Material and Methods Participants}

Patients from the Department of Periodontology, University of Florence, Italy were enrolled for this study, and approved by the local ethical committee. Informed consent was obtained from all subjects to be entered in the study. In obtaining the informed consent and in the conduct of the study, the principles outlined in the Declaration of Helsinki on experimentation involving human subjects were adhered to.

Individuals satisfying the following entry criteria were recruited:

- Age $\geqslant 18$ years.

- No systemic diseases.

- No pregnancy.

- No active periodontal treatment and systemic antibiotic therapy in the last 6 months.

- Presence of at least one incisor, one premolar and one molar in each quadrant.

- Presence of at least two teeth with at least one site with probing depths (PD) that ranged between 4 and $9 \mathrm{~mm}$ in each quadrant with bleeding on probing (BoP).

\section{Sample size}

The sample size was calculated using $\alpha=0.05$ and the power $(1-\beta)=80 \%$. For the variability $(\sigma=\mathrm{SD})$, the value of $0.6 \mathrm{~mm}$ (Sculean et al. 2004) was used considering clinical attachment level (CAL) gain as a variable outcome. The minimum clinically significant value $(\delta)$ considered was $0.5 \mathrm{~mm}$. On the basis of these data, the number of patient required to be enrolled to conduct this study has been calculated as 24 (Dupont \& Plummer 2003). However, considering the possibility of having a certain amount of drop-out patients, the total number of requested patients was 27 .

\section{Interventions}

\section{Operator training}

A training session for the use of Er:YAG laser device was organized. For this reason, a total of 10 patients were treated preliminarily by an operator with more than 10 years of experience in periodontology (R.R.).

\section{Investigator training}

One examiner (F.C.) different from the operator was required to attend a training and calibration session on five periodontal patients aimed at: (1) instruction and calibration in the measurement techniques to be used; (2) instruction in the compilation of the data collection sheets and (3) preliminary data recording session. Some clinical variables, such as pocket depth, gingival recession, CAL, plaque index (presence/absence) and BoP (presence/absence), were measured twice after an interval of $1 \mathrm{~h}$ in order to evaluate the reliability of the examiner using the intra-class correlation coefficient $(\rho)$.

\section{Treatment phase I: instruction of oral hygiene procedures}

After having entered into the study, all patients received the following procedures by the same operator (J.M.):

- Oral hygiene instructions and appropriated motivation.

- A questionnaire, using a visual analogue scale (VAS) graded from 0 to $100 \mathrm{~mm}$, to evaluate his/her subjective condition such as dental hypersensitivity, pain and chewing discomfort. The patients were asked to place a mark in the appropriate position on the line. The distance from 0 (beginning of the line) was then measured with a millimetre ruler.

- Full-mouth supragingival professional prophylaxis using ultrasonic/ hand-instruments.

\section{Measurements}

One week after the professional oral hygiene instruction and motivation phase, the patients were recalled to collect clinical data. Clinical measurements were performed at baseline, 3 and 
6 months by the same-blinded examiner to the treatment (F.C.).

\section{Clinical measurements}

At baseline (0), 3 and 6 months the following clinical measurements were taken by the same investigator:

- PD at six sites for each tooth (three buccal and three palatal).

- BoP at six sites for each tooth, using the Ainamo \& Bay's Index (1975).

- Plaque index (PII) at six sites for each tooth, using the Ainamo \& Bay's Index (1975).

- Recession depth (Rec) at six sites for each tooth.

- CAL of the six sites per tooth will be calculated as PD+Rec (Rec is equal to 0 whenever the CEJ is covered).

- Full-mouth plaque/bleeding score.

\section{Treatment phase II: non-surgical procedure}

After the data collection, the patients were recalled to undergo treatment phase II. The four quadrants of the mouth were randomized in order to receive one of the following selected treatments by the same operator (R.R.) at the same appointment:

Treatment 1. A supragingival professional prophylaxis $(S)$ : using mechanical instruments (ultrasonics, hand instruments, polishing) was performed. For ethical reasons, this control quadrant was treated at the end of the study as needed.

Treatment 2. Er:YAG+scaling and root planing $(L+S R P)$ : A combination of an Er:YAG laser and a mechanical SRP was performed. In particular, mechanical SRP was performed after the laser application in order to leave the root surface as smooth as possible at the end of treatment.

Treatment 3. Er:YAG laser (L): An Er:YAG device (Smart 2940 Plus, DEKA M.E.L.A. srl, Calenzano, Firenze, Italy) with an energy level of 150 $\mathrm{mJ} /$ pulse and a repetition rate of $10 \mathrm{~Hz}$ (wave length $2.94 \mu \mathrm{m}$ ) was used under water irrigation according to the manufacturer's instruction. A conic fibre tip of $0.5 \mathrm{~mm}$ of diameter (at the point) $\times 10 \mathrm{~mm}$ of length was adopted. The application of the laser treatment was performed from coronal to apical direction (Schwarz et al. 2003a, b) with an inclination of the fibre tip of about $20^{\circ}$ (Folwaczny et al. 2001).
Treatment 4. Mechanical SRP: Gracey's curettes and an ultrasonic device (MiniPiezon ${ }^{\circledR}$, EMS Electro Medical Systems S.A., Nyon, Switzerland) were used.

Local anaesthesia was performed only if needed.

For all treatments, the instrumentation was carried out until the operator felt a planed and well-debrided dental surface. Subgingival root planing and/or laser application were performed in the sites with a periodontal $\mathrm{PD} \geqslant 4 \mathrm{~mm}$. The sites with a $\mathrm{PD}<4 \mathrm{~mm}$ were treated using ultrasonic device and hand instrument/polishing.

The time of execution for each treatment procedure was recorded.

In case of periodontal abscess, the operator re-treated the affected sites by $\mathrm{SRP}$, irrespective of the procedure randomly assigned for that quadrant.

\section{Post-treatment instructions}

All patients were instructed to discontinue toothbrushing for the day of the treatment, avoiding trauma at the treated sites; from the second until the seventh day, tooth cleaning by toothbrush and interproximal instruments were prescribed. A 60 -s rinse with $0.12 \%$ chlorhexidine digluconate was prescribed twice a day for 1 week.

Patients were recalled for control and supportive periodontal therapy (and prophylaxis as needed) at week 1, 4, 12 and 24 weeks by a different operator (J.M.).

\section{Patient evaluation of the operative procedure}

After explaining the post-operative instructions, a second evaluation questionnaire was provided to the patients by the same operator (J.M.). Discomfort or pain perceived during the procedures was recorded by the patient immediately after the end of treatment using a VAS graded from 0 to $100 \mathrm{~mm}$ and referred to each quadrant. One week later, a third questionnaire was provided to the patients to record discomfort or pain perceived during chewing activity, job and daily-life interference and dental hypersensitivity.

Complications, such as fever, periodontal abscess and tooth fracture were also recorded.

At 6 months, benefits of the procedures were again subjectively evaluated by administering a fourth questionnaire. The following data were evaluated using the same visual analogue scale: subjec- tive changes in pain, chewing comfort and dental sensitivity.

\section{Statistical and methodological methods}

Descriptive statistic analysis was performed using frequency and percentage for the qualitative variables, while mean and standard deviation were computed for the quantitative variables. Only sites with $\mathrm{PD} \geqslant 4 \mathrm{~mm}$ at baseline were considered eligible, and therefore included for the analysis.

The statistical analysis was intention to treat. In particular, if a patient showed up at the 3-month recall visit and not at the 6-month re-evaluation, the clinical data were imputed to the 6month analysis. On the contrary, if a patient showed up at the 6-month recall visit and not at the 3-month re-evaluation, the clinical data were imputed to the 3-month analysis. No analysis was performed on patients who did not show up at any recall visits. The same approach was adopted for the extracted teeth.

A restricted maximum likelihood (ReML) method for fitting mixed model was performed. The model considered was full factorial at two factors: Er:YAG laser and mechanical SRP. The site was considered as the statistical unit. The primary outcome variable was the CAL gain and the value of PD at baseline was used in the analysis as a covariate. This model considered the sites clustered into the patients. The interaction $\mathrm{L} \times \mathrm{SRP}$ was also considered in this model. At the beginning, the interactions PD0 $\times \mathrm{L}$, $\mathrm{PDO} \times \mathrm{SRP}$ and $\mathrm{PD} 0 \times \mathrm{L} \times \mathrm{SRP}$ were considered in the analysis but, if not significant, they were successively dropped out from the model.

Other variables (secondary variables) were also considered and analysed using ANOVA test blocked for each patient:

1. Chair-time per treatment (min.).

2. Pain perceived during the treatment execution (VAS).

3. Pain perceived, chewing discomfort and dental hypersensitivity 1 week after treatment (VAS).

4. Pain perceived, chewing discomfort and dental hypersensitivity 6 months after treatment (VAS).

In case of significant results, a post hoc comparison using the Tukey-Kramer honestly significant difference test was performed. 
All the statistical analyses were carried out using the same software (JMP ${ }^{\mathbb{R}} 7.0$ 2007 Copyright ${ }^{\circledR}$ SAS Institute Inc., SAS Campus Drive, Cary, NC, USA).

\section{Randomization and allocation concealment}

In each subject, dental quadrants were randomly assigned to one of the four treatment regimens. Treatment assignment was noted in the registration and treatment assignment form that was kept by the central registrar (M.N., statistician). Allocation concealment was performed by opaque sealed envel- opes, sequentially numbered. The central registrar generated the allocation sequence by means of a computergenerated random permuted block (patient) and instructed a different subject (D.F., secondary investigator) to assign a sealed envelope containing the treatments of each quadrant. The randomization opaque envelope was opened immediately before the beginning of the treatment phase II.

\section{Blinding (masking)}

The examiner was maintained blinded for the treatment.

\section{Results}

Experimental population and calibration of the examiner

A total of 27 patients enrolled between September 2005 and March 2008 participated in the study, but 26 were included in the analyses (Fig. 1, flow-chart). Twenty-four patients completed the study reaching the end of the follow-up period at 6 months. Two patients skipped one measurement appointment: one patient (aged 82 years) did not show up at the 6-month recall visit due to health problems, and the second one underwent the 6-month measurements but skipped the

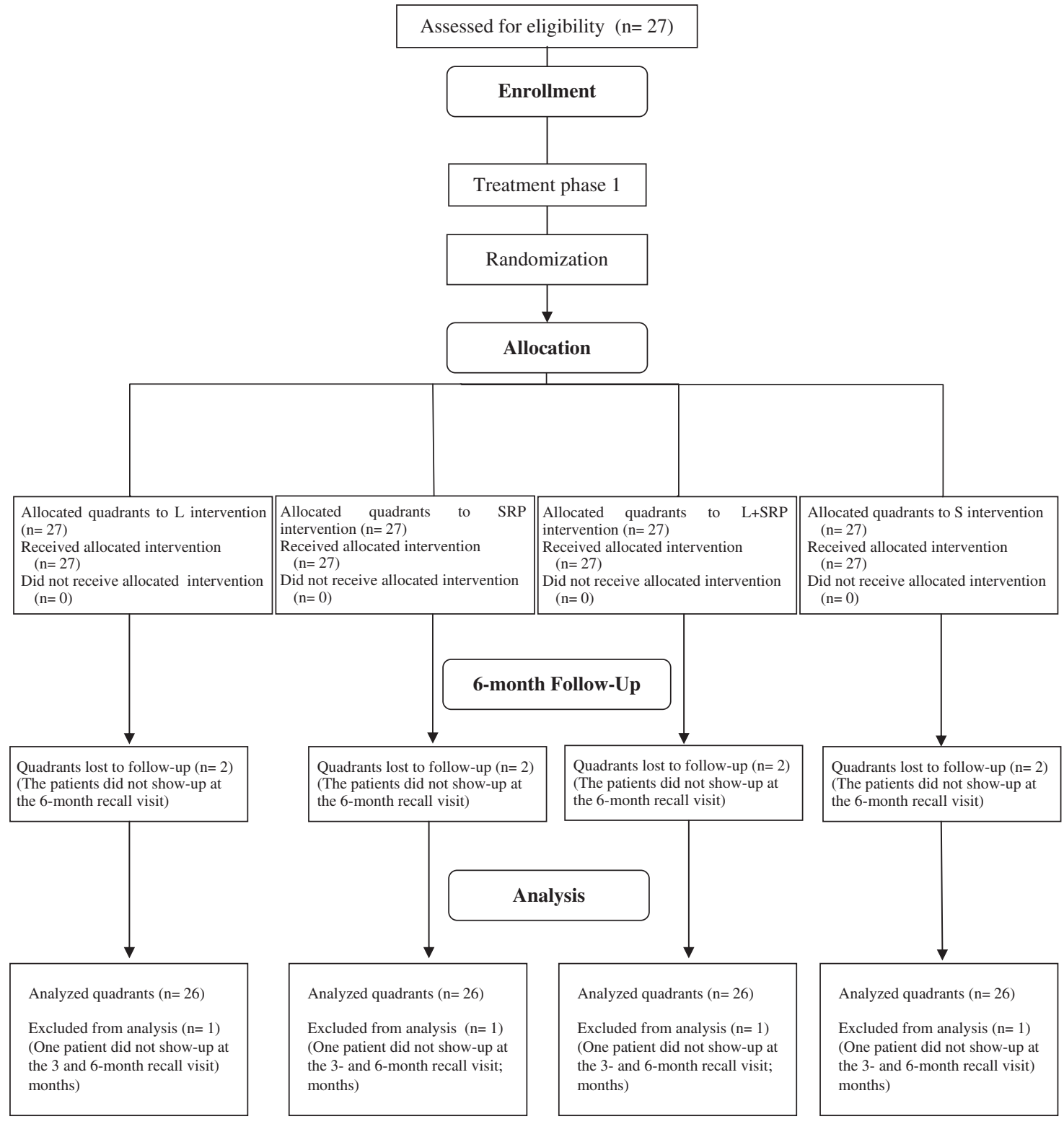

Fig. 1. The CONSORT flow-chart. 
3-month appointment due to his job activities. Both these patients were considered in the analyses. On the contrary, one patient did not show up at the recall visit of 3 and 6 months since he moved away for his job, and therefore was not included in the analysis.

Furthermore, two out of 708 analysed teeth (belonging to the L group) were lost due to excessive hyper-mobility. The sites of one tooth were excluded from the analysis as it was extracted between the baseline and 3-month examination, while the sites of the second tooth were considered in the analysis, as it was lost between the 3- and 6-month examination. The number of analysed sites resulted in a different between the baseline (1671), and the 3- and 6-month recall visit (1582).

The results of the examiner calibration are reported in Table 1 . In particu-

Table 1. Examiner intra-class coefficient correlation calculated after the calibration session

\begin{tabular}{lc}
\hline Variables & Reliability \\
\hline Probing depth (PD) & $0.98^{*}$ \\
Recession (Rec) & $0.96^{*}$ \\
Clinical attachment level (CAL) & $0.97^{*}$ \\
Bleeding on probing (BoP) & $0.89^{\dagger}$ \\
Plaque index (PlI) & $0.91^{\dagger}$ \\
\hline
\end{tabular}

*Intra-class correlation coefficient $(\rho)$.

${ }^{\dagger} k$-statistics. lar, the intra-class correlation coefficient $(\rho)$ of 0.97 resulted for the measurement of the primary outcome variable CAL.

\section{Patient characteristic at baseline}

The mean age of the patients was $50.5 \pm 11.7$ years and 18 out of 27 were females. Twelve patients were smokers $(<10$ cigarettes/day). The clinical measurements recorded in the sites with $\mathrm{PD} \geqslant 4 \mathrm{~mm}$ at baseline for the four treatment groups are reported in Table 2.

Dental hypersensitivity, gingival bleeding, and pain reported by the patients through the questionnaire are enlisted in Table 3.

\section{Immediately at the end of treatment}

Regarding the pain perceived during the treatment procedures and recorded immediately at the end of treatment, the only statistically significant difference resulted between SRP and $S$ $(p=0.0132)$ (Table 3$)$. The chair-time resulted statistically different results among all treatments $(p=0.0001)$. The lowest mean chair-time was recorded for the $S$ group $(6.6 \pm 2.0 \mathrm{~min}$.), followed by the $\mathrm{L}$ group $(12.6 \pm 3.4 \mathrm{~min}$.), the SRP group $(16.2 \pm 5.6 \mathrm{~min}$.) and the $\mathrm{L}+\mathrm{SRP}$ group (19.2 $\pm 6.1 \mathrm{~min}$.).

Table 2. Measurements at baseline calculated on 27 patients (1671 sites with $\mathrm{PD} \geqslant 4 \mathrm{~mm}$ )

\begin{tabular}{lcccc}
\hline & $\begin{array}{c}\text { S group } \\
\text { Sites no. }=387\end{array}$ & $\begin{array}{c}\text { L+SRP group } \\
\text { Sites no. }=419\end{array}$ & $\begin{array}{c}\text { L group } \\
\text { Sites no. }=443\end{array}$ & $\begin{array}{c}\text { SRP group } \\
\text { Sites no. }=422\end{array}$ \\
\hline PD & $5.3 \pm 1.3$ & $5.1 \pm 1.1$ & $5.2 \pm 1.2$ & $5.2 \pm 1.2$ \\
Rec & $0.8 \pm 1.1$ & $0.6 \pm 0.9$ & $0.9 \pm 1.2$ & $0.8 \pm 1.1$ \\
CAL & $6.1 \pm 1.5$ & $5.7 \pm 1.5$ & $6.2 \pm 1.8$ & $6.1 \pm 1.6$ \\
BoP & $288(74 \%)$ & $299(71 \%)$ & $334(75 \%)$ & $309(73 \%)$ \\
PII & $277(72 \%)$ & $264(63 \%)$ & $282(64 \%)$ & $285(68 \%)$ \\
\hline
\end{tabular}

$\mathrm{PD}$, probing depth in $\mathrm{mm}$; BoP, bleeding on probing; PII, plaque index; Rec, gingival recession in $\mathrm{mm}$; CAL, clinical attachment level in $\mathrm{mm}$; SRP, scaling and root planing.

\section{One week after treatment}

After 1 week, no difference was observed for pain $(p=0.5968)$ and chewing discomfort $(p=0.3151)$ between the four types of treatments. However, the SRP procedure caused a higher dental hypersensitivity than the $\mathrm{S}$ treatment $(p=0.0294)$ (Table 3).

\section{Three months after treatment}

Table 4 shows the measurements of the variables PD, BoP, PII, Rec and CAL recorded 3 months after therapy. The variation of the variables CAL, PD and Rec has been considered as CAL gain, $\mathrm{PD}$ gain and Rec reduction (Rec red). These clinical measurements revealed that the L+SRP, L and SRP groups showed a greater CAL gain value $(0.5 \pm 1.6 ; \quad 0.5 \pm 1.7 \quad$ and $\quad 0.6 \pm$ $1.6 \mathrm{~mm}$, respectively) than the $\mathrm{S}$ group $(0.2 \pm 1.6 \mathrm{~mm})$. The PD gain values results were quite similar in groups $\mathrm{L}+\mathrm{SRP}, \mathrm{L}$ and $\mathrm{SRP} \quad(1.1 \pm 1.5$, $0.9 \pm 1.5$ and $1.0 \pm 1.4 \mathrm{~mm})$ with respect to the $S$ group $(0.5 \pm 1.6 \mathrm{~mm})$, while Rec red values were lower in the $\mathrm{L}+\mathrm{SRP}$ group $(-0.6 \pm 1.0 \mathrm{~mm})$ than the other three groups.

\section{Six months after treatment}

Descriptive statistics at 6 months after therapy is reported in Table 5. The variation of the variables CAL, PD and Rec has been considered as CAL gain, PD gain and Rec red.

It is possible to observe that the variable plaque index showed a continuous decrease along with the follow-up time in all the four treatment procedures.

Regarding the variable CAL gain 6 months after therapy, the L+SRP and SRP groups showed a greater CAL gain

Table 3. Questionnaires referred to each quadrant (treatment group) at baseline, end of treatment, 1 week and 6 months

\begin{tabular}{|c|c|c|c|c|c|c|}
\hline Time & Variable & $\mathrm{S}$ group & $\mathrm{L}+\mathrm{SRP}$ group & L group & SRP group & $p$-value \\
\hline \multirow[t]{3}{*}{ Baseline } & Dental hypersensitivity* & $18.7 \pm 30.2$ & $19.9 \pm 33.9$ & $19.7 \pm 29.6$ & $19.3 \pm 30.7$ & - \\
\hline & Gingival bleeding & $3(11 \%)$ & $6(22 \%)$ & $7(26 \%)$ & $10(37 \%)$ & - \\
\hline & Pain* & $10.3 \pm 25.5$ & $7.2 \pm 20.9$ & $2.4 \pm 10.7$ & $3.4 \pm 12.4$ & - \\
\hline End of treatment & Pain* & $13.7 \pm 21.3$ & $30.4 \pm 30.6$ & $21.1 \pm 26.4$ & $34.8 \pm 36.5$ & 0.0132 \\
\hline \multirow{3}{*}{1 week } & Pain* & $13.9 \pm 27.2$ & $13.1 \pm 25.3$ & $17.3 \pm 28.8$ & $20.7 \pm 34.1$ & 0.5968 \\
\hline & Dental hypersensitivity* & $7.6 \pm 19.6$ & $18.0 \pm 26.2$ & $15.1 \pm 27.2$ & $24.2 \pm 31.1$ & 0.0294 \\
\hline & Chewing discomfort* & $8.3 \pm 19.7$ & $7.9 \pm 20.4$ & $16.4 \pm 27.7$ & $11.4 \pm 24.3$ & 0.3151 \\
\hline \multirow[t]{3}{*}{6 months } & Pain* & $15.4 \pm 30.8$ & $10.6 \pm 26.0$ & $6.3 \pm 17.9$ & $10.7 \pm 24.6$ & 0.4732 \\
\hline & Dental hypersensitivity* & $8.4 \pm 22.1$ & $13.5 \pm 26.2$ & $4.4 \pm 15.6$ & $7.1 \pm 17.5$ & 0.3135 \\
\hline & Chewing discomfort* & $10.6 \pm 26.2$ & $8.4 \pm 20.3$ & $10.3 \pm 22.6$ & $9.2 \pm 20.1$ & 0.9574 \\
\hline
\end{tabular}

*VAS values from 0 to 100 .

$p$-value calculated by ANOVA test. 
value $(0.5 \pm 1.7$ and $0.5 \pm 1.8 \mathrm{~mm}$, respectively) than the $\mathrm{S}(0.1 \pm 1.9 \mathrm{~mm})$ and the $\mathrm{L}$ groups $(0.2 \pm 1.9 \mathrm{~mm})$.

The PD gain values resulted greater in $\mathrm{L}+\mathrm{SRP}$ group $(1.5 \pm 1.6 \mathrm{~mm})$, followed by SRP group $(1.0 \pm 1.5 \mathrm{~mm})$, $\mathrm{L}$ and $\mathrm{S}$ groups $(0.7 \pm 1.6$ and $0.7 \pm$ $1.7 \mathrm{~mm}$, respectively).

Regarding the Rec red, the results of $\mathrm{S}$ group $(-0.5 \pm 1.2 \mathrm{~mm})$ showed similar values to the $\mathrm{L}+\mathrm{SRP}, \mathrm{L}$ and SRP groups $\quad(-0.7 \pm 1.1 ; \quad-0.5 \pm 1.2$; $-0.5 \pm 1.1 \mathrm{~mm}$, respectively).

Regarding the patient-centred analysis based on the questionnaire, the variables pain $(p=0.4732)$, dental hypersensitivity $(p=0.3135)$ and chewing discomfort $(p=0.9574)$, did not show any statistically significant difference among the procedures (Table 3 ).

The inferential statistical analyses (ReML) are reported in Tables 6 and 7. In particular, in Table 6 , the model explains $19 \%$ of the total variance. The sites treated with SRP showed a greater CAL gain than the sites treated without SRP ( $p$ value $=0.0026$ ). At the same time, the sites treated with Er:YAG laser showed a greater CAL gain than the sites treated without Er:YAG laser, but the difference was not statistically significant $(p$-value $=0.2077) . \quad$ The interaction $\mathrm{L} \times \mathrm{SRP}$ resulted as statistically not significant, indicating that the combined use of Er:YAG laser+SRP did not show a significant adjunctive benefit with respect to the SRP treatment. Meanwhile, the interactions $\mathrm{PD} 0 \times \mathrm{L}$, $\mathrm{PD} 0 \times \mathrm{SRP}$ and $\mathrm{PD} 0 \times \mathrm{L} \times \mathrm{SRP}$ resulted as statistically not significant (and therefore were not included in the analysis), indicating that there was no difference between the treatments when the PD value changed. However, considering all the treatments, the results showed that the greater the PD0, the greater the CAL gain.

Table 7 shows the comparisons between the different treatments and the differences in terms of CAL gain and confidence intervals (CI) at $95 \%$. On average, 6 months after therapy, the differences with $\mathrm{L}$ and $\mathrm{S}$ treatment, $\mathrm{L}$ and SRP treatments, SRP $+\mathrm{L}$ and $\mathrm{L}$ treatments, $\mathrm{SRP}+\mathrm{L}$ and SRP treatments were not significant. However, SRP treatment showed greater CAL gain values than the $\mathrm{S}$ group $(0.37 \mathrm{~mm}$; $95 \%$ CI $0.05 ; 0.68)$. As well, the $\mathrm{L}+\mathrm{SRP}$ treatment resulted in a greater CAL gain values than the $\mathrm{S}$ group (0.42 mm; 95\% CI $0.11 ; 0.73)$.

Table 4. Clinical outcomes at 3 months calculated on 26 patients (1582 sites with PD $\geqslant 4 \mathrm{~mm}$ )

\begin{tabular}{lcccr}
\hline & $\begin{array}{c}\text { S group } \\
\text { Sites no. }=365\end{array}$ & $\begin{array}{c}\text { L+ SRP group } \\
\text { Sites no. }=405\end{array}$ & $\begin{array}{c}\text { L group } \\
\text { Sites no. }=413\end{array}$ & $\begin{array}{r}\text { SRP group } \\
\text { Sites no. }=399\end{array}$ \\
\hline PD & $4.8 \pm 1.8$ & $4.0 \pm 1.5$ & $4.4 \pm 1.7$ & $4.3 \pm 1.6$ \\
Rec & $1.1 \pm 1.3$ & $1.2 \pm 1.2$ & $1.3 \pm 1.4$ & $1.2 \pm 1.3$ \\
CAL & $5.8 \pm 1.9$ & $5.2 \pm 1.8$ & $5.7 \pm 2.2$ & $5.5 \pm 2.0$ \\
PD gain & $0.5 \pm 1.6$ & $1.1 \pm 1.5$ & $0.9 \pm 1.5$ & $1.0 \pm 1.4$ \\
Rec red & $-0.3 \pm 0.9$ & $-0.6 \pm 1.0$ & $-0.4 \pm 1.0$ & $-0.4 \pm 1.1$ \\
CAL gain & $0.2 \pm 1.6$ & $0.5 \pm 1.6$ & $0.5 \pm 1.7$ & $0.6 \pm 1.6$ \\
BoP & $226(62 \%)$ & $208(51 \%)$ & $242(59 \%)$ & $221(55 \%)$ \\
PII & $201(55 \%)$ & $193(48 \%)$ & $206(50 \%)$ & $200(50 \%)$ \\
\hline
\end{tabular}

$\mathrm{PD}$, probing depth in $\mathrm{mm}$; BoP, bleeding on probing; PII, plaque index; Rec, gingival recession in $\mathrm{mm}$; CAL, clinical attachment level in $\mathrm{mm}$; Rec red, recession reduction in $\mathrm{mm}$; PD gain, variation of probing depth values; CAL gain, variation of clinical attachment level values.

Table 5. Clinical outcomes at 6 months calculated on 26 patients ( 1582 sites with PD $\geqslant 4 \mathrm{~mm}$ )

\begin{tabular}{lcccr}
\hline & $\begin{array}{c}\text { S group } \\
\text { Sites no. }=365\end{array}$ & $\begin{array}{c}\text { L+ SRP group } \\
\text { Sites no. }=405\end{array}$ & $\begin{array}{c}\text { L group } \\
\text { Sites no. }=413\end{array}$ & $\begin{array}{r}\text { SRP group } \\
\text { Sites no. }=399\end{array}$ \\
\hline PD & $4.6 \pm 1.9$ & $3.9 \pm 1.5$ & $4.5 \pm 1.9$ & $4.3 \pm 1.7$ \\
Rec & $1.3 \pm 1.6$ & $1.3 \pm 1.2$ & $1.5 \pm 1.4$ & $1.3 \pm 1.3$ \\
CAL & $5.9 \pm 2.2$ & $5.2 \pm 1.8$ & $6.0 \pm 2.4$ & $5.6 \pm 2.0$ \\
PD gain & $0.7 \pm 1.7$ & $1.2 \pm 1.6$ & $0.7 \pm 1.6$ & $1.0 \pm 1.5$ \\
Rec red & $-0.5 \pm 1.2$ & $-0.7 \pm 1.1$ & $-0.5 \pm 1.2$ & $-0.5 \pm 1.1$ \\
CAL gain & $0.1 \pm 1.9$ & $0.5 \pm 1.7$ & $0.2 \pm 1.9$ & $0.5 \pm 1.8$ \\
BoP & $232(64 \%)$ & $216(53 \%)$ & $239(58 \%)$ & $227(57 \%)$ \\
PII & $182(50 \%)$ & $189(47 \%)$ & $172(42 \%)$ & $191(48 \%)$ \\
\hline
\end{tabular}

$\mathrm{PD}$, probing depth in mm; BoP, bleeding on probing; PII, plaque index; Rec, gingival recession in $\mathrm{mm}$; CAL, clinical attachment level in $\mathrm{mm}$; Rec red, recession reduction in $\mathrm{mm}$; PD gain, variation of probing depth values; CAL gain, variation of clinical attachment level values.

Table 6. Inferential statistics. Restricted maximum likelihood (ReML) method for fitting mixed model. Outcome variable: clinical attachment level gain at 6 months.

\begin{tabular}{lrcrr}
\hline Factor & Estimate & Standard error & $t$-ratio & $p$-value \\
\hline Intercept & -1.44 & 0.26 & -5.58 & $<0.0001$ \\
L & 0.15 & 0.12 & 1.26 & 0.2077 \\
SRP & 0.37 & 0.12 & 3.02 & 0.0026 \\
L $\times$ SRP & -0.10 & 0.17 & -0.58 & 0.5588 \\
PD0 & 0.30 & 0.04 & 7.96 & $<0.0001$ \\
\hline
\end{tabular}

$R^{2}=0.19$; root mean square error $=1.65$; overall mean $=0.32$; number of observations $=1582$; percent of total intra-patient variance $=16 \%$.

\section{Complications}

Five periodontal abscesses were observed throughout the follow-up period and complained by the patients. In this case, these sites were treated by SRP, irrespective of the procedure randomly assigned for that quadrant. Two abscesses arose in one patient, one in S and one in L+SRP treatment group; three other abscesses were observed in three different patients, and in particular two in the $\mathrm{S}$ group and one in the $\mathrm{L}+\mathrm{SRP}$ group. Two teeth were lost during the follow-up period belonging to the $\mathrm{L}$ group. One patient reported fever during the first week after treatment, another patient lost 1 day of work, and another patient reported 1 day of daily-life interference.
Table 7. Least square mean difference between groups using Tukey-Kramer honestly significant difference test

\begin{tabular}{lcrc}
\hline Comparisons & Difference & \multicolumn{2}{c}{ CI 95\% } \\
& & \multicolumn{2}{c}{ min; max) } \\
\hline L+SRP versus S & 0.42 & 0.11 & 0.73 \\
SRP versus S & 0.37 & 0.05 & 0.68 \\
L+SRP versus L & 0.27 & -0.03 & 0.57 \\
SRP versus L & 0.21 & -0.09 & 0.52 \\
L versus S & 0.15 & -0.16 & 0.46 \\
L+SRP versus SRP & 0.05 & -0.25 & 0.36 \\
\hline
\end{tabular}

SRP, scaling and root planning; $\mathrm{L}+\mathrm{SRP}$, erbiumdoped: yttrium-aluminium-garnet + SRP.

\section{Discussion}

The present study was aimed to investigate the efficacy of Er:YAG laser 
compared with the conventional mechanical non-surgical debridement according to the CONSORT statements. The choice of using the CONSORT guidelines was dictated due to the controversial results of the laser application in the treatment of periodontitis reported in the literature. In fact, the CONSORT statements represent a guideline to improve the transparency and reporting of the specific design (i.e. RCT) that was developed for (Moher et al. 2001).

The methodological choice of this study (started in 2005) found confirmation in two recent systematic reviews (Karlsson et al. 2008, Schwarz et al. 2008), which stated that no meta-analysis could be possibly to performed due to the heterogeneity of the selected studies, and reporting the inconsistency of the efficacy of laser treatment as an adjunct to nonsurgical periodontal treatment.

Twenty-seven patients affected by moderate to advanced chronic periodontitis were consecutively enrolled for this study at the public Department of Periodontology, University of Florence. The patients showed a high level of plaque accumulation and BoP and needed periodontal causal therapy. In order to be enrolled in the study, each subjects had to show the presence of at least two teeth with at least one site with PD ranging between 4 and $9 \mathrm{~mm}$ in each quadrant of the mouth. Only sites with a pocket depth $\geqslant 4 \mathrm{~mm}$ were included in the analysis because of SRP, which is one of the selected approaches, is indicated in these conditions (Cobb 1996). The conventional mechanical debridement was compared with the Er:YAG laser because the current literature indentified this laser as the most appropriate device for the non-surgical periodontal treatment (Aoki et al. 2004).

The four different treatment approaches (SRP, SRP+laser, laser and supragingival scaling) provided six inferential statistical comparisons (Tables 6 and 7). Significant statistical differences were found comparing SRP+Laser versus supragingival scaling, where the mean CAL gain was greater in the SRP+laser group than the $\mathrm{S}$ group (difference $0.42 \mathrm{~mm} ; 95 \%$ CI $0.11 ; 0.73$ ), and comparing SRP versus supragingival scaling, where the mean CAL gain was greater in the SRP group than in the $\mathrm{S}$ group (difference $0.37 \mathrm{~mm}$; 95\% CI, 0.05; 0.68). On the other hand, the remaining comparisons did not show significant differences between the groups.
In other words, Er:YAG laser resulted in a similar CAL gain with respect to SRP alone. However, Er:YAG laser did not show an adjunctive benefit when used in conjunction with the SRP alone. Similar results have been reported by Sculean et al. (2004) and Tomasi et al. (2006), comparing Er:YAG laser and ultrasonic instrumentations. In another study, Schwarz et al. (2001), it was observed that the combined treatment Er:YAG laser in conjunction with SRP did not appear to additionally improve the outcome of the therapy compared with Er:YAG laser alone.

On the contrary, Crespi et al. (2007) observed in a 2-year follow-up RCT that the difference between the two experimental groups (laser versus ultrasonic instrumentation) was statistically significant in terms of CAL gain and PD reduction. At the same time, Schwarz et al. (2001, 2003a, b), in two randomized controlled studies reported a significantly higher improvement in terms of CAL gain in the laser-treated sites than in the SRP group.

The results of the questionnaire used in this study reported that, immediately at the end of treatment, the pain perceived by the patients during the different treatment procedures resulted as significantly higher for the sites treated with SRP than the $\mathrm{S}$ group procedure (Table 4). This result was confirmed by another study by Derdilopoulou et al. (2007), but it was in contrast with Tomasi et al.'s study (2006), in which the laser group showed a lower discomfort than the ultrasonic scaling procedure.

Regarding the chair-time factor, the present study revealed a significant difference among all groups, and in particular, the Er:YAG laser group resulted as less time consuming than the SRP group (Table 4). Similar observations were reported by Tomasi et al. (2006).

In conclusion, the results of this RCT reveal some advantages and disadvantages in the use of Er:YAG laser in non-surgical periodontal therapy. The main advantages indicate that (i) Er:YAG laser provides a similar CAL gain value to the one reported by the SRP approach; (ii) it does not cause a significant discomfort for the patient and (iii) it is less time consuming than the other treatments.

However, (i) Er:YAG laser efficacy resulted similar to the supragingival scaling, while the SRP approach resulted in a statistically greater efficacy than the supragingival scaling; (ii) if used in addition to SRP, it does not provide a further adjunctive benefit with respect to SRP alone.

\section{References}

Ainamo, J. \& Bay, I. (1975) Problems and proposals for recording gingivitis and plaque. International Dental Journal 25, 229-235.

Aoki, A., Sasaki, K. M., Watanabe, H. \& Ishikawa, I. (2004) Lasers in nonsurgical periodontal therapy. Periodontology 2000 36, 59-97.

Bower, R. C. (1979) Furcation morphology relative to periodontal treatment. Furcation root surface anatomy. Journal of Periodontology 50, 366-374.

Cobb, C. M. (1996) Non-surgical pocket therapy: mechanical. Annals of Periodontology 1, 443-490.

Crespi, R., Cappare, P., Toscanelli, I., Gherlone, E. \& Romanos, G. E. (2007) Effects of Er:YAG laser compared to ultrasonic scaler in periodontal treatment: a 2-year follow-up split-mouth clinical study. Journal of Periodontology 78, 1195-1200.

Daeveau, R. P., Tanner, A. \& Page, R. C. (1997) The microbial challenge in periodontitis. Periodontology 2000 14, 12-32.

Derdilopoulou, F. V., Nonhoff, J., Neumann, K. \& Kielbassa, A. M. (2007) Microbiological findings after periodontal therapy using curettes, Er:YAG laser, sonic, and ultrasonic scalers. Journal of Clinical Periodontology 34, 588-598.

Dupont, W. D. \& Plummer Jr., W. D. (2003) PS power and sample size calculations, version 2.1.31. Available at http://biostat.mc.vanderbilt. edu/twiki/bin/view/Main/PowerSampleSize.

Folwaczny, M., Mehl, A., Aggstaller, H. \& Hickel, R. (2002) Antimicrobial effects of 2.94 micron Er:YAG laser radiation on root surfaces: an in vitro study. Journal of Clinical Periodontology 29, 73-78.

Folwaczny, M., Thiele, L., Mehl, A. \& Hickel, R. (2001) The effect of working tip angulation on root substance removal using Er:YAG laser radiation: an in vitro study. Journal of Clinical Periodontology 28, 220-226.

Ishikawa, I., Aoki, A. \& Takasaki, A. A. (2004) Potential applications of Erbium:YAG laser in periodontics. Journal of Periodontal Research 39, 275-285.

Israel, M., Cobb, C. M., Rossmann, J. A. \& Spencer, P. (1997) The effects of $\mathrm{CO}_{2}$, Nd:YAG and Er:YAG lasers with and without surface coolant on tooth root surfaces. An in vitro study. Journal of Clinical Periodontology 24, 595-602.

Karlsson, M. R., Diogo Löfgren, C. I. \& Jansson, H. M. (2008) The effect of laser therapy as an adjunct to non-surgical periodontal treatment in subjects with chronic periodontitis: a systematic review. Journal of Periodontology 79, 2021-2028.

Lopes, B. M., Marcantonio, R. A., Thompson, G. M., Neves, L. H. \& Theodoro, L. H. (2008) Short-term clinical and immunologic effects of scaling and root planing with 
Er:YAG laser in chronic periodontitis. Journal of Periodontology 79, 1158-1167.

Moher, D., Schulz, K. F. \& Altman, D. G. (2001) The CONSORT statement: revised recommendations for improving the quality of reports of parallel-group randomised trials. Lancet 357, 1191-1194.

Sanz, W. \& Teughels, W. on behalf of group A of the European Workshop on Periodontology (2008) Innovations in non-surgical periodontal therapy: consensus report of the sixth European workshop on periodontology. Journal Clinical of Periodontology $\mathbf{3 5}$ (Suppl. 8), 3-7.

Schultz, R. J., Harvey, G. P., Fernandez-Beros, M. E., Krishnamurthy, S., Rodriguez, J. E., Cabello, F., Folwaczny, M., Benner, K. U., Flasskamp, B., Mehl, A. \& Hickel, R. (1986) Bactericidal effects of the neodymium:YAG laser: in vitro study. Lasers in Surgery and Medicine 6, 445-448.

Schwarz, F., Aoki, A., Becker, J. \& Sculean, A. (2008) Laser application in non-surgical periodontal therapy: a systematic review. Journal Clinical of Periodontology $\mathbf{3 5}$ (Suppl. 8), 29-44.

Schwarz, F., Sculean, A., Berakdar, M., Georg, T., Reich, E. \& Becker, J. (2003a) Clinical evaluation of an Er:YAG laser combined with scaling and root planing for non-surgical periodontal treatment. A controlled, prospec-

\section{Clinical Relevance}

Scientific rationale for the study: The use of Er:YAG laser therapy has been proposed as an alternative or adjunct to conventional non-surgical therapy. Principal findings: Er:YAG laser did not show a greater efficacy than tive clinical study. Journal of Clinical Periodontology 30, 26-34.

Schwarz, F., Sculean, A., Berakdar, M., Georg, T., Reich, E. \& Becker, J. (2003b) Periodontal treatment with an Er:YAG laser or scaling and root planing. A 2-year follow-up split-mouth study. Journal of Periodontology 74, 590-596.

Schwarz, F., Sculean, A., Georg, T. \& Reich, E. (2001) Periodontal treatment with an Er:YAG laser compared to scaling and root planing. A controlled study. Journal of Periodontology 72, 361-367.

Sculean, A., Schwarz, F., Berakdar, M., Romanos, G. E., Arweiler, N. \& Becker, J. (2004) Periodontal treatment with an Er:YAG laser compared to ultrasonic instrumentation: a pilot study. Journal of Periodontology 75, 966-973.

Slots, J. (1979) Subgingival microflora and periodontal disease. Journal of Clinical Periodontology 6, 351-382.

Slots, J. \& Ting, M. (1999) Actinobacillus actinomycetemcomitans and Porphyromonas gingivalis in human periodontal disease: occurrence and treatment. Periodontology 2000 20, 82-121.

Tewfik, H. M., Garnick, J. J., Schuster, G. S. \& Sharawy, M. M. (1994) Structural and functional changes of cementum surface following exposure to a modified Nd:YAG laser. Journal of Periodontology 65, 297-302.

supragingival scaling and scaling/ root planing, while scaling/root planing showed a greater efficacy than the supragingival scaling in terms of CAL gain. No adjunctive benefit of Er:YAG laser to SRP was observed.
Tomasi, C., Schander, K., Dahlen, G. \& Wennstrom, J. L. (2006) Short-term clinical and microbiologic effects of pocket debridement with an Er:YAG laser during periodontal maintenance. Journal of Periodontology 77, 111-118.

Tunkel, J., Heinecke, A. \& Flemmig, T. F. (2002) A systematic review of efficacy of machine-driven and manual subgingival debridement in the treatment of chronic periodontitis. Journal of Clinical Periodontology 29 (Suppl. 3), 72-81.

Walmsley, A. D., Lea, S. C., Landini, G. \& Moses, A. J. (2008) Advances in power driven pocket/root instrumentation. Journal of Clinical Periodontology 35 (Suppl. 8), 22-28.

Wilder-Smith, P., Arrastia, A. M., Schell, M. J., Liaw, L. H., Grill, G. \& Berns, M. W. (1995) Effect of Nd:YAG laser irradiation and root planing on the root surface: structural and thermal effects. Journal of Periodontology 66, 1032-1039.

Address:

Roberto Rotundo

Department of Periodontology

University of Florence

via Ponte di Mezzo

26-50127, Firenze

Italy

E-mail: roberto.rotundo@unifi.it

Practical implications: Considering the clinical outcome of this study, the clinician also has to take into consideration the high economic cost of the laser machine (when compared with the mechanical instruments). 